\title{
Global Solution of the Generalized Abel Integral Equation by Implicit Interpolation*
}

\author{
By H. Brunner
}

\begin{abstract}
The construction of a (global) approximate solution for a given generalized Abel integral equation may be viewed as a problem of (implicit) interpolation in a prescribed linear space. In this paper, piecewise polynomials (extended spline functions) of a given degree and of class $C$ are used to generate such an approximating function. Results on convergence and error bounds are given, and the practical application of this method is illustrated by a numerical example.
\end{abstract}

1. Introduction. The generalized Abel integral equation has the form

$$
\int_{0}^{x} \frac{G(x, t)}{(x-t)^{\alpha}} y(t) d t=g(x), \quad x \in I=[0, a], \quad 0<\alpha<1 .
$$

It is well known (see, for example, [1, p. 26]) that (1.1) possesses a (unique) solution $y(x) \in C(I)$ if $G(x, t)$ and $g(x)$ satisfy the following conditions (which are assumed to hold throughout this paper):

$$
G(x, t) \in C(T), \quad \partial G(x, t) / \partial x \in C(T),
$$

$$
\text { where } T=\{(x, t): 0 \leqq t \leqq x \leqq a\} .
$$

$$
\begin{gathered}
G(x, x) \neq 0, \quad x \in I . \\
F(x) \equiv \int_{0}^{x} \frac{g(t)}{(x-t)^{1-\alpha}} d t \in C^{1}(I) .
\end{gathered}
$$

Observe that under these conditions the limit

$$
y(0)=\lim _{x \rightarrow 0_{+}}\left(g(x) \cdot x^{\alpha-1}(1-\alpha) / G(0,0)\right)
$$

exists.

Let $N \geqq 1$ and $m \geqq 1$ be given integers, and define the points $\left\{\xi_{k}\right\}$ by $0=\xi_{0}<$ $\xi_{1}<\cdots<\xi_{N}=a$. Let $Z_{N}=\left\{\xi_{k}: k=0,1, \cdots, N-1\right\}$. The exact solution $y(x)$ of (1.1) will be approximated by piecewise polynomials (or extended spline functions) of degree $m$ which are of continuity class $C(I)$ and have the knots $Z_{N}$. We denote this class of functions by $S_{m}{ }^{(0)}\left(Z_{N}\right)$. An element $s \in S_{m}{ }^{(0)}\left(Z_{N}\right)$ has a unique representation of the form

Received November 24, 1972.

AMS (MOS) subject classifications (1970). Primary 65R05.

Key words and phrases. Implicit interpolation, generalized Abel integral equation, continuous approximate solution, piecewise polynomials.

* This research was supported by the National Research Council of Canada (Grant No. A-4805). 


$$
s(x)=p(x)+\sum_{k=1}^{N-1} \sum_{\nu=0}^{m-1} \gamma_{k \cdot \nu} \cdot\left(x-\xi_{k}\right)_{+}^{m-\nu},
$$

where $p(x) \in \pi_{m}$ (see also [3]). Here,

$$
\begin{aligned}
(x-\beta)_{+}^{n} & =(x-\beta)^{n}, & & x \geqq \beta, \\
& =0, & & x<\beta .
\end{aligned}
$$

An approximate solution $s \in S_{m}{ }^{(0)}\left(Z_{N}\right)$ for (1.1) will be found by using an approach which may be regarded as implicit interpolation. To be precise, let

$$
\xi_{k}=x_{k \cdot 0}<x_{k \cdot 1}<\cdots<x_{k \cdot m}=\xi_{k+1}, \quad k=0,1, \cdots, N-1 .
$$

Define the linear functionals $\left\{L_{k \cdot j}\right\}$ by setting

$$
\begin{aligned}
L_{k \cdot j}(f)=\int_{\xi_{0}}^{x_{k \cdot j}} K\left(x_{k \cdot j}, t\right) \cdot f(t) d t \quad & (f \in C(I)), \\
j & =1, \cdots, m ; k=0, \cdots, N-1,
\end{aligned}
$$

with $K(x, t) \equiv G(x, t) /(x-t)^{\alpha}$. We wish to find an element $s \in S_{m}{ }^{(0)}\left(Z_{N}\right)$ such that

$$
L_{k \cdot j}(s)=L_{k \cdot j}(y)=g\left(x_{k \cdot j}\right), \quad j=1, \cdots, m ; k=0, \cdots, N-1,
$$

satisfying

$$
s\left(\xi_{0}\right)=y(0) .
$$

THEOREM 1. Let $G$ and $g$ in (1.1) satisfy the conditions (i), (ii), (iii) above, and assume that $G(x, t) \neq 0$ in $T$. Then there exists a unique $s \in S_{m}{ }^{(0)}\left(Z_{N}\right)$, with $p \in \pi_{0}$, which satisfies the interpolating conditions (1.6).

Proof. Define the functions $\left\{\varphi_{i}(x): i=0, \cdots, m N\right\}$ by

$$
\begin{aligned}
\varphi_{i}(x) & =1, & i & =0, \\
= & \left(x-\xi_{0}\right)_{+}^{i}, & i & =1, \cdots, m, \\
& \vdots & & \\
& =\left(x-\xi_{N-1}\right)_{+}^{i-m(N-1)}, & i & =m(N-1)+1, \cdots, m N .
\end{aligned}
$$

Furthermore, let

$$
\psi_{i}(x)=\int_{\xi_{0}}^{x} K(x, t) \cdot \varphi_{i}(t) d t, \quad i=0, \cdots, m N
$$

We have, by assumption on $G, \psi_{i} \in C(I)$, with $\psi_{i}(x) \equiv 0$ on $\left[\xi_{0}, \xi_{\nu}\right]$ for $i>\nu m$. It is easily seen that these functions $\left\{\psi_{i}(x)\right\}$ are linearly independent on $I$. However, they do not satisfy the Haar condition on $I$ since, for $\alpha_{i}=0, i=0, \cdots, \nu m ; \alpha_{i} \neq 0$, $i>\nu m, \psi(x)=\sum_{i=0}^{m N} \alpha_{i} \psi_{i}(x)$ vanishes identically on [0, $\left.\xi_{\nu}\right]$. On the other hand, since $G(x, t) \neq 0$ in $T$, the functions $\left\{\psi_{i}(x): i=\nu m+1, \cdots,(\nu+1) m\right\}$ do satisfy the Haar condition on the left-open part of $\sigma_{\nu} \equiv\left[\xi_{\nu}, \xi_{\nu+1}\right]$, since any nontrivial function

$$
\sum_{i=\nu m+1}^{(\nu+1) m} \alpha_{i} \psi_{i}(x)=\int_{\xi_{\nu}}^{x} K(x, t) \cdot\left(t-\xi_{\nu}\right) \cdot \sum_{i=\nu m+1}^{(\nu+1) m} \alpha_{i}\left(t-\xi_{\nu}\right)^{i-\nu m-1} d t
$$


has at most $(m-1)$ zeros in $\left(\xi_{v}, \xi_{v+1}\right]$. Hence the linear functionals (1.5) are linearly independent in the conjugate space of $S_{m}{ }^{(0)}\left(Z_{N}\right)$. This implies (see also [4, p. 26]) that for a given value $\alpha_{0}$ there exists a unique set $\left\{\alpha_{1}, \cdots, \alpha_{m N}\right\}$ such that

$$
L_{k \cdot j}\left(\sum_{i=0}^{m N} \alpha_{i} \psi_{i}\right)=g\left(x_{k \cdot j}\right), \quad j=1, \cdots, m ; k=0, \cdots, N-1 .
$$

This completes the proof of Theorem 1 .

The above proof suggests that the unknown coefficients in (1.3) may be computed recursively, using the intervals $\left\{\sigma_{k}: k=0, \cdots, N-1\right\}$. For computational purposes, we shall choose for $s \in S_{m}{ }^{(0)}\left(Z_{N}\right)$ the representation

$$
s(x)=s_{k}(x)=\sum_{\nu=0}^{m} \frac{c_{k \cdot v}}{\nu !}\left(x-\xi_{k}\right)^{\nu}, \quad x \in \sigma_{k},
$$

which is equivalent to (1.3), with $p=c_{0 \cdot 0}$. Since $s \in C(I)$ we have

$$
c_{k, 0}=s_{k-1}\left(\xi_{k}\right), \quad k=1, \cdots, N-1,
$$

and we choose

$$
c_{0.0}=s_{0}\left(\xi_{0}\right)=y(0) \quad \text { (given by (1.2)). }
$$

(We note that another possible representation for $s(x)$ is (1.7) with the functions $\left\{\left(x-\xi_{k}\right)^{\nu}\right\}$ replaced by the Chebyshev polynomials $\left\{T_{\nu}(x)\right\}$ for the interval $\sigma_{k}$. This form is recommended if $m$ is large. Compare also [3].)

The unknown coefficients $\left\{c_{k \cdot v}\right\}$ in (1.7) are now determined recursively by requiring that, for a given $k$,

$$
L_{k \cdot j}(s)=L_{k \cdot j}(y)=g\left(x_{k \cdot j}\right), \quad j=1, \cdots, m,
$$

and by observing the conditions (1.8). Theorem 1 implies that each of the linear systems (1.9) possesses a unique solution $\left\{c_{k \cdot 1}, \cdots, c_{k \cdot m}\right\}, k=0, \cdots, N-1$.

It is clear that Theorem 1 will in general not remain valid if $G(x, t)$ vanishes at some points in $T$ (we have $G(x, t) \not \equiv 0$, by assumption (ii) above). In such cases, the choice of the points $\left\{\xi_{k}\right\}$ and $\left\{x_{k \cdot j}\right\}$ will be governed by the function $G$ under consideration, in order to get a unique solution for (1.9).

Generalized Abel integral equations of the form (1.1) have recently been considered by Weiss [7] and by Weiss and Anderssen [8], who used product integration techniques to generate approximate values to $y(x)$ at given discrete points in $I$. It may be of interest to note here that an idea related to the ones used in product integration and in the approach taken in this paper was introduced by Huber [6] in 1939 to find approximate solutions of linear first-kind Volterra integral equations with continuous kernels.

2. Convergence and Error Bounds. For a given set of knots $Z_{N}$, define

$$
\begin{aligned}
H_{k} & =\xi_{k+1}-\xi_{k}, \quad k=0, \cdots, N-1, \\
H & =\max _{(k)}\left(H_{k}\right), \quad \bar{H}=\min _{(k)}\left(H_{k}\right), \\
\pi_{N} & =H / \bar{H}, \quad N=1,2, \cdots .
\end{aligned}
$$

For simplicity in notation, we shall deal with the case of uniformly spaced points 
$\left\{x_{k \cdot j}\right\}$, i.e., $x_{k \cdot j}=\xi_{k}+j \cdot h_{k}, j=1, \cdots, m$, with $h_{k}=H_{k} / m, k=0, \cdots, N-1$. Define the error function $e(x)$ by $e(x)=s(x)-y(x)$. Clearly, $e \in C(I)$. The approximating function $s \in S_{m}{ }^{(0)}\left(Z_{N}\right)$ shall be given by (1.7).

LEMMA 1. Assume that $y \in C^{m+1}(I)$, and let $B_{k}=\left(\beta_{k \cdot 1}, \cdots, \beta_{k \cdot m}\right)^{T}$ be defined by

$$
c_{k \cdot \nu}=y^{(\nu)}\left(\xi_{k}\right)+\beta_{k, \nu}\left(h_{k}\right)^{m+1-\nu}, \quad \nu=1, \cdots, m ; k=0, \cdots, N-1 .
$$

If $N \rightarrow \infty, H \rightarrow 0$ (with $\xi_{0}=0, \xi_{N}=a$ ) such that $\pi_{N} \leqq \gamma$ for all $N$, then

$$
\left\|B_{k}\right\|_{1}=\sum_{\nu=1}^{m}\left|\beta_{k \cdot \nu}\right| \leqq B \quad \text { for all } k \text {. }
$$

Proof. Let

$$
\varphi_{k \cdot \nu}(x)=\left(x-\xi_{k}\right)^{\nu} / h_{k}^{\nu}, \quad \nu=1, \cdots, m+1 ; k=0, \cdots, N-1 .
$$

For $x \in \sigma_{k}$, we then have

$$
e(x)=e\left(\xi_{k}\right)+h_{k}^{m+1} \cdot\left(\sum_{\nu=1}^{m} \frac{\beta_{k \cdot \nu}}{\nu !} \varphi_{k \cdot \nu}(x)-T_{k}(y) \cdot \varphi_{k \cdot m+1}(x)\right),
$$

where $T_{k}(y)=y^{(m+1)}\left(\eta_{k}(x)\right) /(m+1) !, \xi_{k}<\eta_{k}(x)<x$. By construction of $s(x)$, the error function satisfies

$$
L_{k \cdot j}(e)=0, \quad j=1, \cdots, m ; k=0, \cdots, N-1 .
$$

We proceed by induction: For $k=0$ we obtain

$$
\sum_{\nu=1}^{m} \frac{\beta_{0 \cdot \nu}}{\nu !} \int_{\xi_{0}}^{x_{0} \cdot i} K\left(x_{0 \cdot i}, t\right) \varphi_{0 \cdot v}(t) d t=\int_{\xi_{0}}^{x_{0} \cdot i} K\left(x_{0 \cdot i}, t\right) \cdot T_{0}(y) \cdot \varphi_{0 \cdot m+1}(t) d t
$$

(using $e\left(\xi_{0}\right)=0$; a trivial modification will yield results similar to those below if $\left.e\left(\xi_{0}\right)=\mathcal{O}\left(H^{\alpha}\right), q \geqq 1\right)$.

By definition of the functions $\left\{\varphi_{k \cdot v}(x)\right\}$, and by assumptions on $G, g$, and $y$, the right-hand side of $(2.4)$ is $\mathcal{O}\left(h_{0}{ }^{1-\alpha}\right)$. Furthermore, the matrix with elements

$$
\frac{1}{\nu !} \int_{\xi_{0}}^{x_{0 \cdot j}} G\left(x_{0 \cdot i}, x_{0 \cdot j}\right) \cdot \varphi_{0 \cdot \nu}(t) \cdot\left(x_{0 \cdot j}-t\right)^{-\alpha} d t \quad(j, \nu=1, \cdots, m)
$$

is essentially a Vandermonde matrix and hence nonsingular. A simple calculation yields for these elements the expression

$$
G\left(x_{0 \cdot i}, x_{0 \cdot j}\right) \frac{j^{\nu+1-\alpha} \cdot h_{0}^{1-\alpha}}{(1-\alpha) \cdots(1+\nu-\alpha)},
$$

with $G(x, x) \neq 0, x \in I$. Since $G(x, t) \in C(T)$, there exists a $\delta_{0}>0$ such that for all $h_{0} \in\left(0, \delta_{0}\right)$ the solution of $(2.4)$ satisfies $\beta_{0 . \nu}=\mathcal{O}(1), \nu=1, \cdots, m$. We thus obtain

$$
e\left(\xi_{1}\right)=e\left(\xi_{0}\right)+m h_{0}^{m+1} \sum_{\nu=1}^{m} \frac{\beta_{0 \cdot \nu} \cdot m^{\nu}}{\nu !}+\mathcal{O}\left(h_{0}^{m+1}\right)=\mathcal{O}\left(H_{0}^{m+1}\right),
$$

or, since $\bar{H} \leqq m h_{0} \leqq H$,

$$
e\left(\xi_{1}\right)=\mathcal{O}\left(H^{m+1}\right) \text {. }
$$

Let now $k>0$. It follows from (2.3) and (2.2) that 


$$
\begin{aligned}
h_{k}^{m+1} \sum_{\nu=1}^{m} \frac{\beta_{k \cdot \nu}}{\nu !} \int_{\xi_{k}}^{x_{k \cdot j}} K\left(x_{k \cdot i}, t\right) \varphi_{k \cdot \nu}(t) d t & \\
= & -e\left(\xi_{k}\right) \int_{\xi_{k}}^{x_{k \cdot i}} K\left(x_{k \cdot i}, t\right) d t-\sum_{\mu=0}^{k-1} e\left(\xi_{\mu}\right) \int_{\xi_{\mu}}^{\xi_{\mu+1}} K\left(x_{k \cdot i}, t\right) d t \\
& -\sum_{\mu=0}^{k-1} h_{\mu}^{m+1} \sum_{\nu=1}^{m} \frac{\beta_{\mu \cdot \nu}}{\nu !} \int_{\xi_{\mu}}^{\xi_{\mu+1}} K\left(x_{k \cdot j}, t\right) \varphi_{\mu \cdot \nu}(t) d t+\mathcal{O}\left(H^{m+2-\alpha}\right) .
\end{aligned}
$$

This may be rewritten as

$$
\sum_{\nu=1}^{m} \frac{\beta_{k \cdot \nu}}{\nu !} \int_{\xi_{k}}^{x_{k \cdot j}} G\left(x_{k \cdot i}, t\right) \varphi_{k \cdot \nu}(t) \cdot\left(x_{k \cdot i}-t\right)^{-\alpha} d t=\mathcal{O}\left(H^{1-\alpha}\right) .
$$

Here we have made use of the fact that $h_{\mu} / h_{k} \leqq H / \bar{H}=\pi_{N} \leqq \gamma$ for all $N$, and $k H \leqq$ $N H \leqq \gamma N \hat{H} \leqq \gamma a$. We conclude, by an argument similar to the one used for $k=0$, that there exists a $\delta_{k}>0$ such that for all $h_{k} \in\left(0, \delta_{k}\right)$ the unique solution of (2.5) satisfies $\beta_{k \cdot \nu}=O(1), \nu=1, \cdots, m$, and $k \leqq N$.

THEOREM 2. Under the assumptions of Lemma 1 ,

$$
|e(x)| \leqq \gamma a H^{m}\left(B+M_{m+1}\right), \quad x \in Z_{N} .
$$

Here, $B$ is defined in Lemma 1 , and

$$
M_{m+1}=\max _{x \in I}\left|y^{(m+1)}(x)\right| /(m+1) !
$$

Proof. From (2.2) we find, using the fact that $e \in C(I)$,

$$
\begin{aligned}
\left|e_{k}\left(\xi_{k}\right)\right| & \leqq\left|e_{k-1}\left(\xi_{k-1}\right)\right|+\left(m \cdot h_{k-1}\right)^{m+1}\left(\sum_{\nu=1}^{m} \frac{\left|\beta_{k-1} \cdot \nu\right|}{\nu !}+M_{m+1}\right) \\
& \leqq\left|e_{k-1}\left(\xi_{k-1}\right)\right|+H^{m+1} \cdot\left(B+M_{m+1}\right),
\end{aligned}
$$

where we have set $e_{k}(x)=s_{k}(x)-y(x), x \in \sigma_{k}$. By a well-known result on inequalities of this type (see, for example, [5, p. 18]), we obtain (using again $e\left(\xi_{0}\right)=0$ )

TABLE I

\begin{tabular}{ccccccccc}
\hline$k$ & $\begin{array}{c}x=\xi_{k} \\
(N=90)\end{array}$ & $\begin{array}{c}e(x) \text { for } \\
m=1\end{array}$ & $k$ & $\begin{array}{c}x=\xi_{k} \\
(N=45)\end{array}$ & $\begin{array}{c}e(x) \text { for } \\
m=2\end{array}$ & $k$ & $\begin{array}{c}x=\xi_{k} \\
(N=60)\end{array}$ & $\begin{array}{c}e(x) \text { for } \\
m=3\end{array}$ \\
\hline 1 & 0.2 & $5.07 \cdot 10^{-2}$ & 1 & 0.4 & $-2.65 \cdot 10^{-2}$ & 1 & 0.3 & $1.46 \cdot 10^{-2}$ \\
2 & 0.4 & $-9.68 \cdot 10^{-3}$ & 2 & 0.8 & $-6.18 \cdot 10^{-3}$ & 2 & 0.6 & $-2.01 \cdot 10^{-3}$ \\
3 & 0.6 & $6.68 \cdot 10^{-3}$ & 3 & 1.2 & $-1.41 \cdot 10^{-3}$ & 3 & 0.9 & $4.73 \cdot 10^{-4}$ \\
. & & & & & & & & \\
. & & & & & & & & \\
30 & 6.0 & $7.22 \cdot 10^{-5}$ & 15 & 6.0 & $9.63 \cdot 10^{-6}$ & 20 & 6.0 & $5.67 \cdot 10^{-6}$ \\
. & & & & & & & & \\
. & & & & & & & & \\
. & 12.0 & $2.55 \cdot 10^{-5}$ & 30 & 12.0 & $4.01 \cdot 10^{-6}$ & 40 & 12.0 & $1.99 \cdot 10^{-6}$ \\
\hline 0 & & & & & & & & \\
. & & & & & & & & \\
90 & 18.0 & $1.39 \cdot 10^{-5}$ & 45 & 18.0 & $2.28 \cdot 10^{-6}$ & 60 & 18.0 & $1.08 \cdot 10^{-6}$ \\
\hline
\end{tabular}




$$
\begin{aligned}
\left|e_{k}\left(\xi_{k}\right)\right| & \leqq k H^{m+1}\left(B+M_{m+1}\right) \leqq N H \cdot H^{m} \cdot\left(B+M_{m+1}\right) \\
& \leqq \gamma a H^{m}\left(B+M_{m+1}\right),
\end{aligned}
$$

for we have assumed that the ratio $\pi_{N}=H / \bar{H}$ remains bounded as $N \rightarrow \infty: \pi_{N} \leqq \gamma$. Hence $N H \leqq \gamma N \hat{H} \leqq \gamma a$.

Theorem 2 remains essentially valid if we consider $e(x)$ for $x \notin Z_{N}, x \in I$. We have

THEOREM 3. Under the assumptions of Lemma 1,

$$
|e(x)| \leqq H^{m} \cdot\left(\gamma a\left(B+M_{m+1}\right)+\mathcal{O}(H)\right) \text { for all } x \in I .
$$

Proof. For $x \in \sigma_{k}$ we get, from (2.2) and (2.6),

$$
\begin{aligned}
|e(x)| & \leqq\left|e\left(\xi_{k}\right)\right|+H^{m+1} \cdot\left(\left\|B_{k}\right\|_{1}+M_{m+1}\right) \\
& \leqq H^{m}\left(\gamma a\left(B+M_{m+1}\right)+H\left(B+M_{m+1}\right)\right) \\
& =H^{m}\left(B+M_{m+1}\right) \cdot(\gamma a+H) .
\end{aligned}
$$

We conclude by observing that the degree $m$ of $s(x)$ may be treated as a parameter which may be changed anytime during the computation. Furthermore, the knots $Z_{N}$ need not be chosen a priori but may be selected during the computational process, according to the character of the given equation (1.1) and its exact solution $y(x)$.

3. Numerical Example. We illustrate the application of the method of piecewise polynomials described above by solving the Abel integral equation

\section{TABLE II}

Change of stepsize (spacing of knots) during computation:

Initial spacing: $H_{k}=0.01, k=0, \cdots, 50$.

For $k>50: H_{k}=0.5$.

\begin{tabular}{rcc}
\hline$k$ & $x=\xi_{k}$ & $e(x) \quad(m=2)$ \\
\hline 1 & 0.01 & $-4.19 \cdot 10^{-3}$ \\
2 & 0.02 & $-9.77 \cdot 10^{-4}$ \\
3 & 0.03 & $-2.24 \cdot 10^{-4}$ \\
$\cdot$ & & \\
. & & \\
. & & $3.20 \cdot 10^{-7}$ \\
49 & 0.49 & $3.11 \cdot 10^{-7}$ \\
50 & 0.50 & $-5.81 \cdot 10^{-4}$ \\
51 & 1.00 & $-2.87 \cdot 10^{-4}$ \\
52 & 1.50 & \\
$\cdot$ & & \\
. & & $-3.35 \cdot 10^{-7}$ \\
84 & 17.50 & $-3.12 \cdot 10^{-7}$ \\
85 & 18.00 &
\end{tabular}




$$
\int_{0}^{x} \frac{y(t) d t}{(x-t)^{1 / 2}}=x, \quad 0 \leqq x \leqq 18 .
$$

Its exact solution $y(x)=2 x^{1 / 2} / \pi$ has derivatives which are unbounded at $x=0$.

Equation (3.1) was solved numerically by functions $s \in S_{r}{ }^{(0)}\left(Z_{N}\right)$ for $r=1,2,3$. A selection of numerical results is listed in Table I. Table II shows, for $s \in S_{2}{ }^{(0)}\left(Z_{N}\right)$, how a relatively large change in stepsize (from $H_{k}=0.01$ to $H_{k}=0.5$ ) affects the numerical results.

All the computations were performed on the CDC 6400 (single precision) at Dalhousie University Computer Centre.

Department of Mathematics

Dalhousie University

Halifax, Nova Scotia, Canada

1. M. Bôcher, An Introduction to the Study of Integral Equations, 2nd ed., Cambridge Univ. Press, London, 1914.

2. H. BRUNNER, "The numerical solution of the generalized Abel integral equation by piecewise polynomials," Notices Amer. Math. Soc., v. 19, 1972, p. A-662. (Abstract)

3. M. G. Cox, "Curve fitting with piecewise polynomials," J. Inst. Math. Appl., v. 8, 1971, pp. 36-52. MR 44 \#4870.

4. P. J. DAvis, Interpolation and Approximation, Blaisdell, New York, 1963. MR 28

5. P. HENRICI, Discrete Variable Methods in Ordinary Differential Equations, Wiley, New York, 1962. MR 24 \#B1772.

6. A. HUBER, "Eine Näherungsmethode zur Auflösung Volterrascher Integralgleichungen," Monatsh. Math. Phys., v. 47, 1939, pp. 240-246.

7. R. WEIss, "Product integration for the generalized Abel equation," Math. Comp., v. 26, 1972, pp. 177-190. MR 45 \#8050.

8. R. WEISS \& R. S. ANDERSSEN, "A product integration method for a class of singular first kind Volterra equations," Numer. Math., v. 18, 1972, pp. 442-456. 